\title{
Hypothyroidism and non-cardiogenic pulmonary edema: are we missing something here?
}

\author{
Mohammed Al-Sofiani, Dhimitri Nikolla' and V V S Ramesh Metta \\ Department of Medicine, South Buffalo Mercy Hospital, University at Buffalo, Buffalo, New York, USA \\ 'Lake Erie College of Osteopathic Medicine, Erie, Pennsylvania, USA
}

Correspondence

should be addressed

to M Al-Sofiani

Email

dr_moh2008@hotmail.com

\section{Summary}

We report the case of a 42-year-old female with a history of hypothyroidism and asthma presenting with progressive dyspnea and orthopnea after 2 days of an upper respiratory tract infection (URTI). Based on the clinical and radiological findings, the patient was admitted as a case of cardiogenic pulmonary edema secondary to possible viral myocarditis. However, a normal brain natriuretic peptide (BNP) level with a normal ejection fraction (EF) on echocardiogram changed our working diagnosis from cardiogenic to non-cardiogenic pulmonary edema. Further questioning revealed a history of nocturnal snoring, frequent awakening, and daytime fatigue, suggesting a possible sleep apnea syndrome (SAS). In conclusion, we believe that SAS was the missing link between our patient's hypothyroidism and non-cardiogenic pulmonary edema.

\section{Learning points:}

- Always keep an open mind and look for a pathology that would explain the whole clinical scenario.

- The involvement of the respiratory system in hypothyroidism can range from SAS, pulmonary hypertension, hypoventilation, and severe respiratory failure.

- Hypothyroidism and SAS should be considered in the differential diagnosis of non-cardiogenic pulmonary edema.

- Patients should be instructed to take levothyroxine on an empty stomach 30-60 min before food to avoid erratic absorption of the hormone.

\section{Background}

This case outlines the clinical approach to identifying the etiology of pulmonary edema in the setting of hypothyroidism. Pulmonary edema associated with hypothyroidism has been explained by the loss of inotropic and chronotropic effects of the thyroid hormone in the heart (i.e., cardiogenic pulmonary edema) (1). However, in this report, we demonstrate a case of hypothyroidism associated with non-cardiogenic pulmonary edema explained by a different mechanism. We believe that this case exemplifies the importance of keeping an open mind and looking for a pathology that would explain the whole clinical scenario.

\section{Case presentation}

A 42-year-old female with a past medical history of mild intermittent asthma and hypothyroidism (status after radioactive iodine treatment for Graves' disease 15 years ago) presented to the emergency department (ED) with progressive shortness of breath for 1 week. She had been doing well and not seen her primary care physician for about 5 years. A week before her ED presentation, she developed a sore throat and dry cough for 2 days. Shortly after, she started to have exertional dyspnea, which progressed gradually from New York Heart Association (NYHA) classes II-IV over the course of 4 days. This was associated with significant orthopnea 
causing insomnia the night before her ED presentation. She also noticed swelling of both lower limbs and hands.

Review of systems was remarkable for lethargy, cold intolerance, constipation, menorrhagia for 5 years, nocturnal snoring, frequent awakening, and daytime fatigue for about 4 months. When asked about compliance, the patient insisted that she had been taking levothyroxine $\left(\mathrm{L}-\mathrm{T}_{4}\right)$ everyday with her breakfast, which includes a cup of coffee.

On physical examination, our patient was sitting upright and in moderate respiratory distress. She was afebrile with a blood pressure of $113 / 65 \mathrm{mmHg}$, heart rate of $61 / \mathrm{min}$, respiratory rate of $22 / \mathrm{min}$, oxygen saturation of $98 \%$ on room air, and BMI of 32 . There was no pulsus paradoxus. Heart sounds were regular with no extra-heart sound, murmur, or gallop. Neck circumference was 18 inches with no jugular venous distension (JVD). She had bilateral inspiratory basal crackles with no rhonchi on respiratory examination and bilateral +1 pitting edema in her lower extremities.

Which of the following is the most likely explanation for the shortness of breath in this patient?

i) Asthma exacerbation.

ii) Pulmonary edema.

iii) Pneumonia.

iv) Pulmonary embolism (PE).

Despite the past medical history of asthma in our patient, the absence of rhonchi and the presence of bilateral basal inspiratory crackles make the diagnosis of acute asthma exacerbation less likely. The history of significant orthopnea and findings of peripheral pitting edema and bilateral lung crackles on examination favor the diagnosis of pulmonary edema. Pneumonia is less likely in the absence of fever and sputum production. It is also very unlikely that our patient had a PE, given that she was not hypoxic or tachycardic and had no risk factors for PE. Her calculated Wells' score of 0 makes PE very unlikely.

Given the timeline of the symptoms and the acute presentation of orthopnea a few days after upper respiratory tract infection, our most likely diagnosis was cardiogenic pulmonary edema possibly secondary to viral myocarditis and non-ischemic cardiomyopathy.

\section{Investigation}

Chest X-ray (CXR) showed pulmonary edema (Fig. 1).

Which of the following tests would you order next?

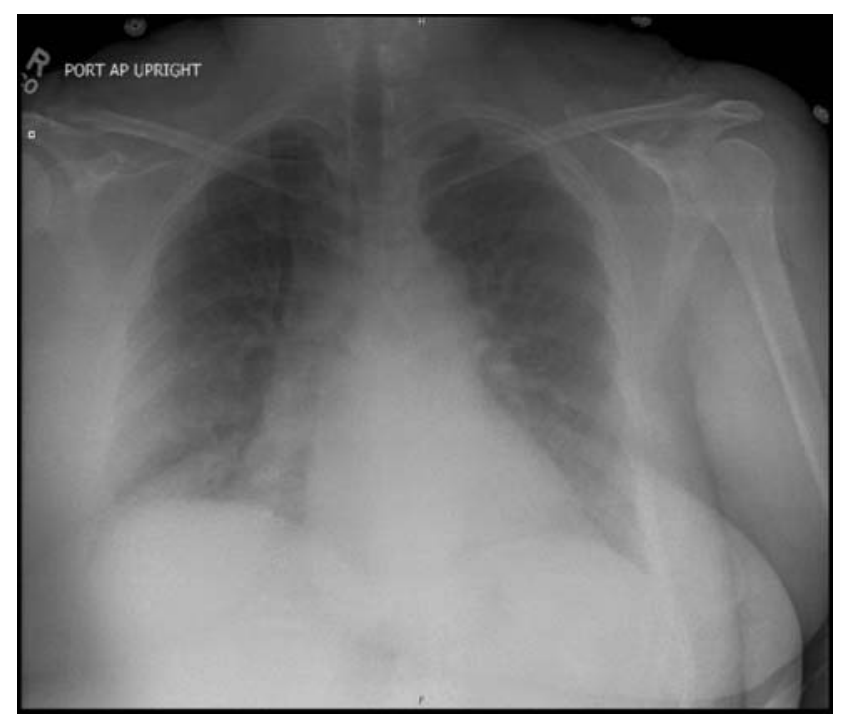

Figure 1

Chest X-ray showing pulmonary edema.

i) D-dimer.

ii) Brain natriuretic peptide.

iii) Polysomnography.

iv) Angiocardiography.

The plasma level of BNP can be measured in the ED and utilized as a simple and reliable biomarker to differentiate cardiogenic pulmonary edema from non-cardiogenic pulmonary edema (2). Our patient had a BNP level of $16 \mathrm{pg} / \mathrm{ml}$ making congestive heart failure and cardiogenic pulmonary edema very unlikely. The results of her remaining blood tests are outlined in Table 1 including a thyrotropin (TSH) level of $43.72 \mathrm{mIU} / \mathrm{l}$ and $\mathrm{T}_{4}<0.2 \mathrm{mIU} / \mathrm{l}$.

Electrocardiogram (EKG) showed sinus bradycardia (heart rate $(\mathrm{HR})=55 / \mathrm{min}$ ) with a first-degree heart block and no acute ST or T wave changes. Echocardiogram showed an ejection fraction of 60-65\% with normal diastolic function and mildly elevated right ventricular systolic pressure at $44.7 \mathrm{mmHg}$. Computed tomography of the chest showed ground-glass densities predominant in the lung-dependent regions compatible with pulmonary edema and no PE (Fig. 2).

\section{Treatment}

At this stage, our working diagnosis changed from cardiogenic to non-cardiogenic pulmonary edema. The patient was started on furosemide $40 \mathrm{mg}$ intravenously daily for 2 days and the $\mathrm{L}_{-} \mathrm{T}_{4}$ dose was increased from 175 to $225 \mu \mathrm{g}$. The patient was advised to take $\mathrm{L}-\mathrm{T}_{4}$ first in the morning on an empty stomach. 
Table 1 Results of the initial blood tests.

\begin{tabular}{l}
\hline Test \\
\hline WBC \\
Hemoglobin \\
Mean corpuscular volume (MCV) \\
Platelets \\
BUN \\
Creatinine \\
eGFR \\
TSH \\
Free T $_{4}$ \\
B-type natriuretic peptide (BNP) \\
Cholesterol \\
LDL \\
HDL \\
Triglyceride \\
Anti-neutrophil antibodies (ANA) \\
Anti-double-stranded DNA antibody \\
(anti ds-DNA) \\
Complement 3 \\
Complement 4
\end{tabular}

\begin{tabular}{l} 
Result \\
\hline $8.2\left(\times 10^{3}\right)$ \\
$8.7(\mathrm{~g} / \mathrm{dl})$ \\
$80(\mathrm{fl})$ \\
427 \\
$12(\mathrm{mg} / \mathrm{dl})$ \\
$1.40(\mathrm{mg} / \mathrm{dl})$ \\
$57.83(\mathrm{ml} / \mathrm{min}$ per 1.73$)$ \\
$43.72(\mathrm{mlU} / \mathrm{l})$ \\
$<0.20(\mathrm{ng} / \mathrm{dl})$ \\
$16(\mathrm{pg} / \mathrm{ml})$ \\
$401(\mathrm{mg} / \mathrm{dl})$ \\
$332(\mathrm{mg} / \mathrm{dl})$ \\
$55(\mathrm{mg} / \mathrm{dl})$ \\
$70(\mathrm{mg} / \mathrm{dl})$ \\
Positive (homogeneous) \\
$9(\mathrm{lU} / \mathrm{ml})$ \\
$125(\mathrm{mg} / \mathrm{dl})$ \\
$28(\mathrm{mg} / \mathrm{dl})$
\end{tabular}

\section{Outcome and follow-up}

Over the course of 2 weeks, the shortness of breath and orthopnea subsided gradually and by the fourth night, the patient was able to sleep on one pillow with no orthopnea. Two weeks later, her shortness of breath, orthopnea, and lower extremity edema resolved and furosemide was discontinued. Four weeks later, her TSH level dropped to $0.37 \mathrm{mIU} / 1$. She declined the sleep study and wanted to be treated only for hypothyroidism.

\section{Discussion}

Identifying the underlying etiology of the first episode of pulmonary edema can sometimes be challenging as all causes of pulmonary edema share the same clinical features to some extent. The first step in approaching pulmonary edema is to differentiate cardiac etiologies from non-cardiac etiologies. Clinical features that point toward cardiogenic pulmonary edema include history of acute coronary syndrome or congestive heart failure and the finding of S3, JVD, or peripheral pitting edema on physical examination. Other features of cardiogenic pulmonary edema include elevated cardiac enzymes and enlarged cardiac silhouette or the presence of Kerley B lines in CXR (3). In contrast, non-cardiac etiologies of pulmonary edema should be suspected in patients with ongoing pulmonary or non-pulmonary infection and sleep apnea syndrome (SAS), those at the risk of aspiration pneumonia or inhalation injury, and after multiple blood transfusions (3). The gold standard test to differentiate cardiogenic pulmonary edema from non-cardiogenic pulmonary edema is pulmonary artery catheterization (4). However, this invasive procedure is rarely used in clinical practice except for patients who are in shock with pulmonary edema of unclear etiology. The production of BNP by the cardiac ventricles in response to ventricular expansion makes it a good biomarker to differentiate between cardiogenic and non-cardiogenic pulmonary edema. BNP level $<100 \mathrm{pg} / \mathrm{ml}$ has a negative predictive value of $>90 \%$, while BNP level of $>500 \mathrm{pg} / \mathrm{ml}$ has a positive predictive value of $>90 \%$ (5).

Severe hypothyroidism can lead to congestive heart failure and cardiogenic pulmonary edema through the loss of inotropic and chronotropic effects of thyroid hormone (1). However, on rare occasions such as our patient's case, severe hypothyroidism can indirectly lead to non-cardiogenic pulmonary edema. SAS is believed to be the missing link between hypothyroidism and non-cardiogenic pulmonary edema in most of these cases (6). The association between hypothyroidism and central or obstructive SAS has been described in the literature (7). Proposed mechanisms of this association include deposition of mucoprotein in the upper airway causing airway obstruction and obstructive SAS (7). More interestingly, thyroid replacement was found to be effective in treating both hypothyroidism and SAS in reported cases (8) (9). Another explanation of pulmonary edema is that capillary permeability increases in state of hypothyroidism, which subsequently results in the leakage of plasma proteins (10).

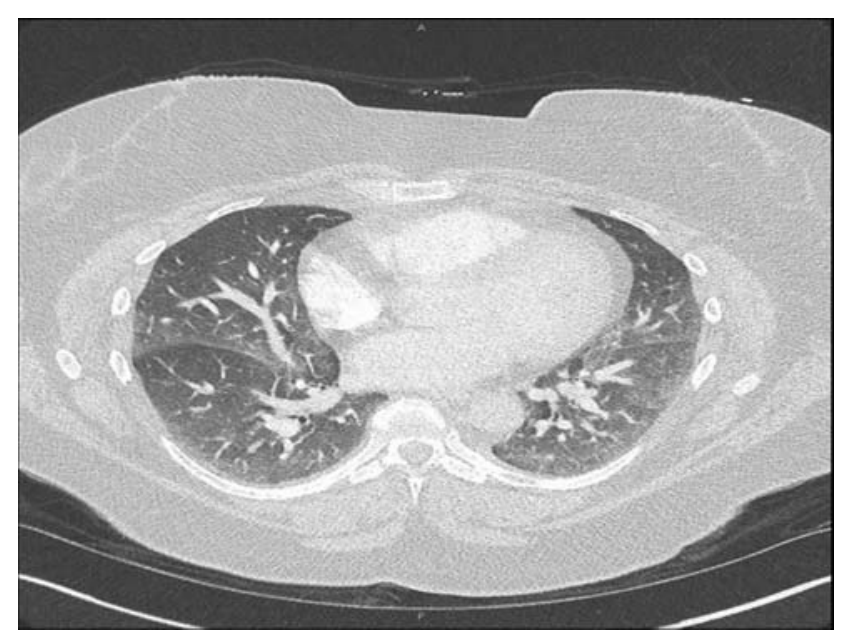

Figure 2

Computed tomography of the chest showing ground-glass densities compatible with pulmonary edema. 


\section{Declaration of interest}

The authors declare that there is no conflict of interest that could be perceived as prejudicing the impartiality of the research reported.

\section{Funding}

This research did not receive any specific grant from any funding agency in the public, commercial or not-for-profit sector.

\section{Patient consent}

Written informed consent was obtained from the patient for publication.

\section{Author contribution statement}

M Al-Sofiani was responsible for patient care during the hospital stay and prepared the manuscript. D Nikolla and R Metta were responsible for patient care and reviewed the manuscript.

\section{References}

1 Klein I \& Danzi S 2007 Thyroid disease and the heart. Circulation 116 1725-1735. (doi:10.1161/CIRCULATIONAHA.106.678326)
2 Januzzi JL, Jr, Camargo CA, Anwaruddin S, Baggish AL, Chen AA, Krauser DG, Tung R, Cameron R, Nagurney JT, Chae CU et al 2005 The N-terminal Pro-BNP investigation of dyspnea in the emergency department (PRIDE) study. American Journal of Cardiology 95 948-954. (doi:10.1016/j.amjcard.2004.12.032)

3 Perina DG 2003 Noncardiogenic pulmonary edema. Emergency Medicine Clinics of North America 21 385-393. (doi:10.1016/S0733-8627(03) 00020-8)

4 Matthay MA \& Chatterjee K 1988 Bedside catheterization of the pulmonary artery: risks compared with benefits. Annals of Internal Medicine 109 826-834. (doi:10.7326/0003-4819-109-10-826)

5 Schreiber D, Schraga ED, Natriuretic peptides in congestive heart failure. http://emedicine.medscape.com/article/761722-overview. updated: January 10, 2012 (accessed Januray 2015)

6 Lopez A, Lorente JA, Jerez V \& Julia JA 1990 Sleep apnea, hypothyroidism and pulmonary edema. Chest 97 763-764. (doi:10.1378/chest. 97.3.763b)

7 Kittle WM \& Chaudhary BA 1988 Sleep apnea and hypothyroidism. Southern Medical Journal 81 1421-1425. (doi:10.1097/00007611198811000-00020)

8 Lin CC, Tsan KW \& Chen PJ 1992 The relationship between sleep apnea syndrome and hypothyroidism. Chest 102 1663-1667. (doi:10.1378/ chest.102.6.1663)

9 Coffernils M, Ravez P, Rivière-Lorfèvre A, Herbaut C, Coulon JP \& Robience Y 1989 Sleep apnea syndrome and hypothyroidism: apropos of a new case and a review of the literature. Revue Médicale de Bruxelles 10 89-93.

10 Lange K 1944 Capillary permeability in myxedema. American Journal of Medical Sciences 208 5-15. (doi:10.1097/00000441-194407000-00002)

Received in final form 25 February 2015

Accepted 23 March 2015 